\title{
Target Detection in Bistatic Radar Sea Clutter Using Stationary Wavelet Transforms
}

\author{
Vichet Duk*, Luke Rosenberg*十, Brian $\mathrm{Ng}^{*}$, \\ Matthew Ritchie ${ }^{\ddagger}$, Riccardo Palamà $\grave{~}^{\S}$ and Hugh Griffiths ${ }^{\S}$ \\ ${ }^{*}$ University of Adelaide, Australia, ${ }^{\dagger}$ Defence Science and Technology Group, Australia, \\ ${ }^{\ddagger}$ Defence Science and Technology Laboratory, UK, ${ }^{\S}$ University College London, UK \\ Email:vichet.duk@adelaide.edu.au
}

Keywords: Sea clutter, bistatic radar, detection, wavelets.

\begin{abstract}
Radar backscatter from the sea-surface is typically nonstationary and may contain sea-spikes which resemble targets and can cause false detections in a radar system. To improve detection performance, this paper investigates a technique based on stationary wavelet transforms (SWT) and demonstrates its performance on both monostatic and bistatic seaclutter data. By exploiting the different components (subbands) of the SWT decomposition, this technique can better distinguish between the different spectral components of the returned signal. The detection performance is measured by Monte Carlo simulation with a cell averaging constant false alarm rate (CA-CFAR) detector. The most appropriate choice of sub-band is selected using an a entropy based metric.
\end{abstract}

\section{Introduction}

Small target detection in the maritime environment remains a challenging problem when the sea-clutter is strong and returns from breaking waves resemble targets [1]-[3]. A potentially useful approach to improve detection performance is to apply signal processing methods prior to detection. Existing examples include coherent processing [1], [4], time-frequency processing, [5] and space-time adaptive processing [6].

The NetRAD multistatic radar system was jointly developed by the University College London in the UK and the University of Cape Town in South Africa [7]. Since the multistatic trials in 2010, researchers have extensively analysed the $\mathrm{Ne}$ tRAD data to gain better understanding of the clutter behaviour [8]-[10] and develop multistatic coherent detection techniques [11]. This analysis showed that the bistatic mean reflectivity and amplitude statistics vary with the bistatic angle, typically having a lower mean backscatter and being less spiky than the monostatic data [8], [9].

There have been a number of investigations into the use of wavelet transforms (WT) for detecting targets in sea-clutter. Two methods were proposed in [12] to improve the signal to noise ratio (SNR) of simulated radar data in Gaussian noise.
The first approach used a wavelet function with an optimal scale as a matched filter, while the second integrated a small range of wavelet coefficients around the optimal scale. This second approach improved robustness and was shown to improve the SNR even further. Ball and Tolley [13] proposed an automatic algorithm to determine the optimal scale parameter, which required searching for the peak in the continuous WT coefficients, followed by a de-noising procedure around the optimum scale. They achieved an average SNR improvement of up to $10 \mathrm{~dB}$ when compared to a conventional matched filter. Zhang et al. [14] also implemented WTs for radar target detection and found that eliminating some coefficients of the wavelet 'detail' sub-band was effective in reducing the noise in radar echoes. By combining this method with independent component analysis, further detection improvement was achieved. However, with limited experimental results, it is unclear whether this approach is effective in general.

In previous work, a novel detection technique using stationary wavelet transforms (SWT) has been presented and applied to sea-clutter collected from an airborne platform [15]. In this paper, we demonstrate the robustness of this technique for both monostatic and bistatic sea-clutter collected by the NetRAD radar system. This paper is organised as follows. Section 2 gives descriptions of NetRAD trial and data analysis. The background on SWTs and the detection scheme is briefly described in Section 3. Section 4 then illustrates the detection performance with the final conclusions given in Section 5 .

\section{NetRAD data}

\subsection{NetRAD system and trial}

NetRAD is a ground-based pulsed-Doppler coherent multistatic radar system consisting of three identical nodes [7]. For the sea-clutter collections, the radar operated with two nodes triggered using GPS disciplined oscillators and synchronised through a $5 \mathrm{GHz}$ wireless link [16]. The radar independently recorded both horizontal and vertical polarisations with a centre frequency of $2.4 \mathrm{GHz}$, a $45 \mathrm{MHz}$ bandwidth, $1 \mathrm{kHz}$ pulse repetition frequency (PRF) and a peak power of $400 \mathrm{~W}$. 
The data was recorded during a series of trials in October 2010 on the coast of South Africa near the Cape Point area. The setup of the trial is shown in Fig. 1 with one node transmitting and two receiving. Data was collected for three bistatic angles, $\beta$ of $60^{\circ}, 90^{\circ}$ and $120^{\circ}$ with a baseline that ran Northwest to Southeast and a separation between the two nodes of approximately $1827 \mathrm{~m}$. The wind direction was from the North with a speed that increased during the trials from $10.2 \mathrm{~m} / \mathrm{s}$ to $12.3 \mathrm{~m} / \mathrm{s}$, while the wave height varied between $3.28 \mathrm{~m}$ to $4.2 \mathrm{~m}$. This corresponds to a Douglas sea state 5 . Both nodes were looking cross-wind with the waves travelling towards the receiver of the bistatic node.

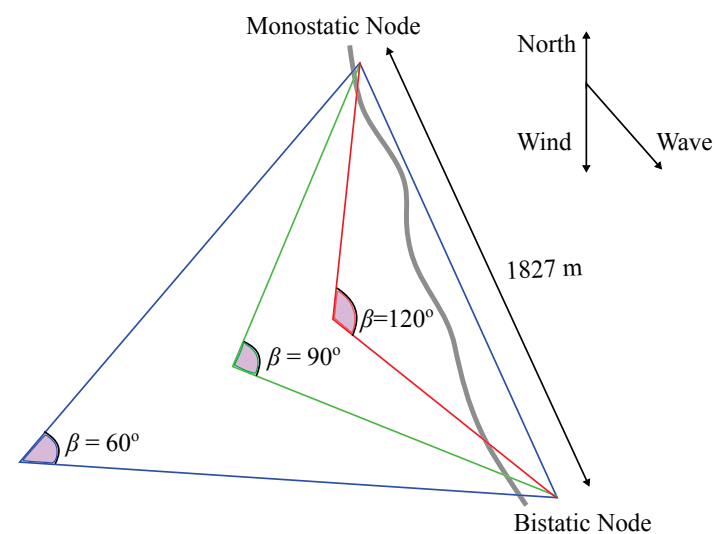

Fig. 1: Illustration of NetRAD trial for three bistatic angles: $60^{\circ}, 90^{\circ}$ and $120^{\circ}$ [17].

\subsection{NetRAD data analysis}

Understanding the characteristics of the NetRAD sea clutter is important to determine the correct wavelet decomposition level for the SWT detection scheme. Figures 2-3 show examples of the horizontally polarised monostatic and bistatic data recorded at the 'bistatic' look angle of $60^{\circ}$ with the time domain on top, the normalised Doppler spectrum or power spectral density (PSD) in the middle and the average PSD on the bottom. The data consists of strong returns that vary greatly from range bin to range bin. Some of these last for short periods of time while others persist over the whole observation time. These 'sea-spikes' are the result of breaking waves and can easily be confused with persistent targets.

A detailed study of the mean Doppler spectrum over all monostatic and bistatic collections has revealed $3 \mathrm{~dB}$ widths between $10-20 \mathrm{~Hz}$ and centre frequencies of up to $\pm 50 \mathrm{~Hz}$. Figure 4 shows the average monostatic (top) and bistatic (bottom) PSD over range with blue, red dash and black dashdot lines representing the bistatic angles, $\beta=60^{\circ}, 90^{\circ}$ and $120^{\circ}$ respectively. These spectra appear quite Gaussian with the monostatic results spread slightly more than the bistatic results. In general there is little difference between them and also between the different bistatic angles. Note that the monostatic data collected at $\beta=90^{\circ}$ contains a small peak at approximately $80 \mathrm{~Hz}$. This appears in only a few range bins and resembles a point target-like component. This could possibly be an extremely fast wave or a recreational boat in the area at the time of the trials. To investigate the component further, Fig. 5 show an example of the horizontally polarised monostatic and bistatic PSD for the 'bistatic' look angle of $90^{\circ}$. This figure confirms that the component exists in both mono and bistatic configurations and is travelling toward the monostatic receiver.
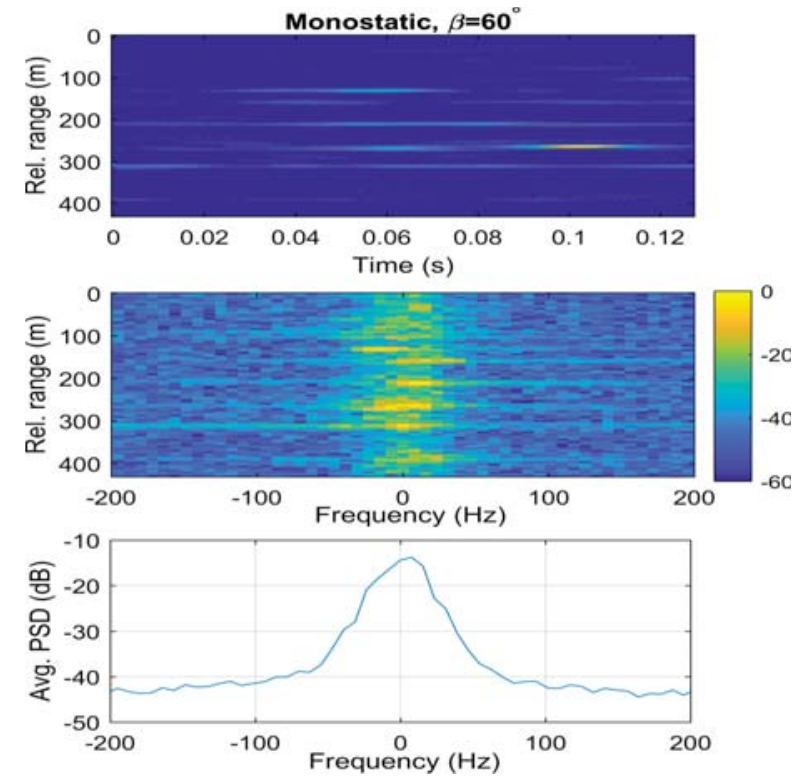

Fig. 2: NetRAD horizontally polarised monostatic data at $\beta=60^{\circ}$ : (top) time domain, (middle) PSD, (bottom) averaged PSD.
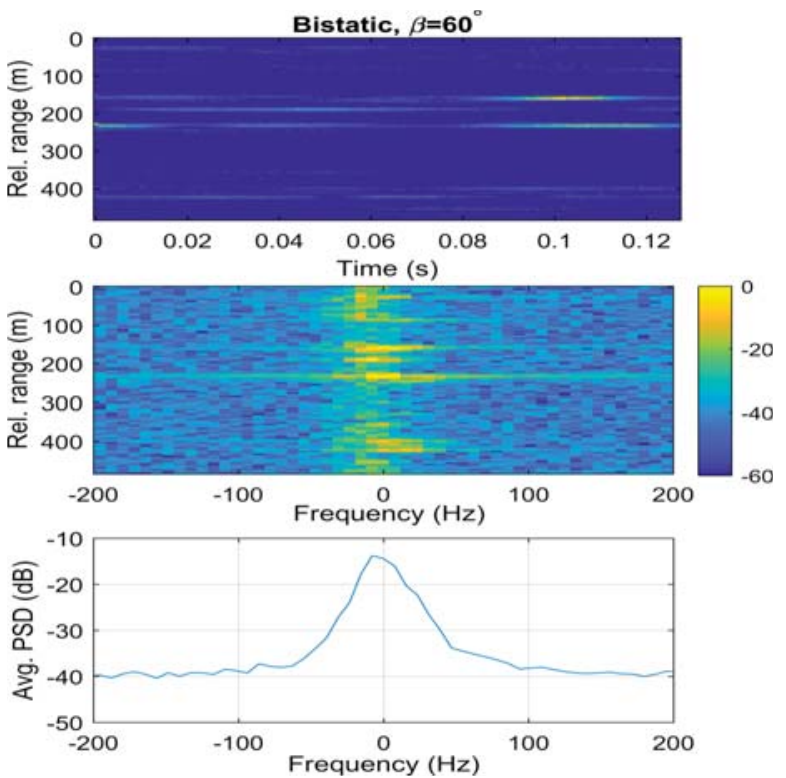

Fig. 3: NetRAD horizontally polarised bistatic data at $\beta=60^{\circ}$ : (top) time domain, (middle) PSD, (bottom) averaged PSD. 

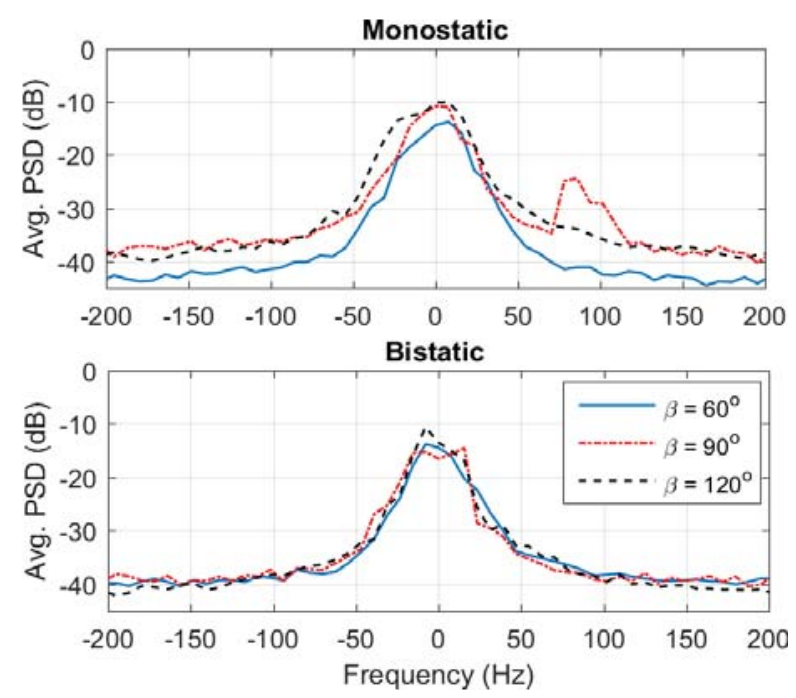

Fig. 4: Average PSD for both monostatic and bistatic configurations with horizontal polarisation.
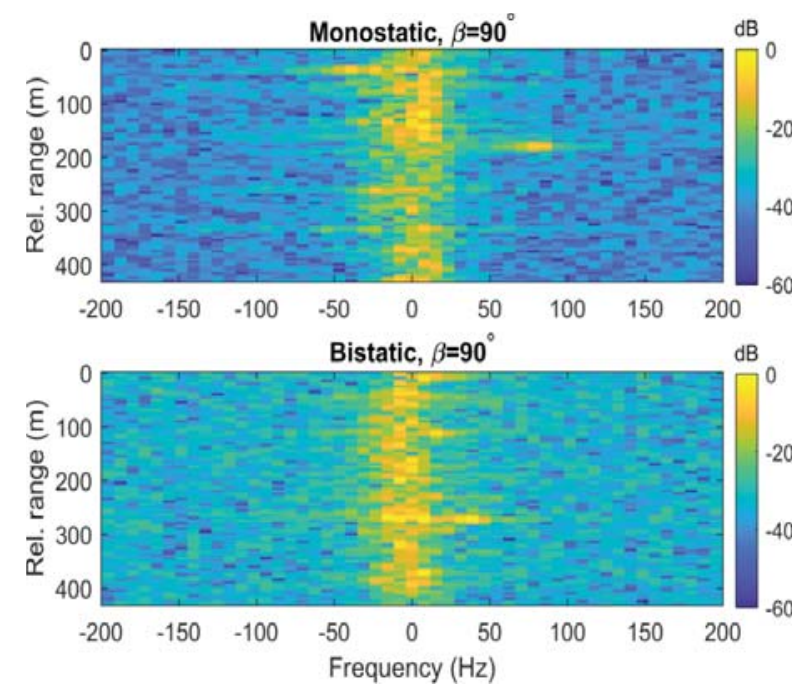

Fig. 5: NetRAD horizontally polarised PSD at $\beta=90^{\circ}$ : (top) monostatic (bottom) bistatic.

\section{Wavelet based detection scheme}

Full details of the SWT detection scheme and its use on the Ingara medium grazing angle data are provided in [15]. In this section, a brief summary is provided, along with some modifications to apply the scheme to NetRAD data.

\subsection{Stationary wavelet transforms}

Wavelet transforms (WT) are joint time-frequency representations commonly used for the analysis of non-stationary signals. They offer a different partitioning of the time frequency plane compared to techniques such as short time Fourier Transforms [18]. The analysing functions in WT are dilated (by a factor a) and shifted (in time by $b$ ) versions of the same wavelet function $\psi(t)$,

$$
\psi_{a, b}(t)=\frac{1}{\sqrt{a}} \psi\left(\frac{t-b}{a}\right) .
$$

Multi-resolution analysis separates a signal into different sets of detail (projections onto wavelet functions, $\psi_{a, b}(t)$ ) and approximate (projections onto scaling function) coefficients, called sub-bands. Each of these sub-bands occupy a unique region of the time-frequency plane.

In this work, we use a variant of the WT called the stationary wavelet transform (SWT), chosen for its translation-invariance property [19]. The SWT signal decomposition algorithm is implemented as cascaded two-channel filter banks, each consisting of a pair of complementary analysis low-pass and high-pass filters. The filter bank at level $k>0$ accepts the approximate sub-band at level $k-1$ as an input and produces detail $\left(D_{k}\right)$ and approximate $\left(A_{k}\right)$ sub-bands at the outputs of the high- and low-pass filters, respectively. The original data is equivalent to the $A_{0}$ sub-band. In computing the SWT, the maximum level of decomposition, $K$, is usually a user-chosen parameter. In the SWT decomposition, the filter impulse responses are up-sampled by 2 at every level of decomposition. This causes rapid growth in the length of these filters as the level $(k)$ increases, eventually reaching the length of the data series. In our case, the finite number of pulses places a practical ceiling on this value and further decomposition beyond this ceases to produce meaningful subbands. If there are $M$ samples in slow-time, and the maximum length of the analysis filters is $N$, then $K$ must satisfy

$$
K \leq \log _{2}(M / N)
$$

and the level $k=1,2, \ldots, K$. A comprehensive survey of the performance of different wavelets is beyond the scope of this paper. Instead, the popular Daubechies-4 wavelet has been chosen for the analysis of the radar backscatter, based on earlier work [20]. With a coherent processing interval (CPI) of $M=128$ pulses and a filter length $N=8$ for the Daubechies4 filter, the SWT analysis is therefore limited to 4 levels or lower as indicated in (2).

\subsection{Sub-band isolation}

The different time-frequency characteristics of the interference and target implies that some reconstructed sub-bands have a stronger signal to interference ratio (SIR) than the original data. Therefore, with careful selection of these sub-bands, we can utilise the reconstructed data to improve the target detection. In the frequency domain, this can be interpreted as band-pass filtering.

The exact combination of sub-bands needs to be carefully determined for different radar systems. Factors that influence the sub-band choice include the PRF and the Doppler bandwidth of the received signal. For the NetRAD data, the PRF is $1 \mathrm{kHz}$ and the clutter spectrum has $3 \mathrm{~dB}$ widths in the range of $10-20 \mathrm{~Hz}$ with a maximum frequency shift of up to 
$\pm 50 \mathrm{~Hz}$. For SWT processing, half of the Doppler spectrum is maintained in the approximate sub-band while the other half is maintained in the detail sub-band. Therefore, to reach the edge of the NetRAD clutter spectrum given a typical frequency shift, the SWT must decompose the signal at least 3 times to reduce the spectral width to $62.5 \mathrm{~Hz}$ (i.e. $500 / 2^{3}$ ). In this case, the first reconstruction will produce the approximate, $\tilde{A}_{3}$ and detail, $\tilde{D}_{123}$ reconstructed sub-bands where $\tilde{D}_{123}$ is a combination of detail sub-bands $D_{1}, D_{2}$ and $D_{3}$. If the target is embedded in the centre of the Doppler endo-clutter region, a final decomposition of the $A_{3}$ sub-band can be performed. Once reconstructed, this gives two new sub-bands: $\tilde{A}_{4}$ and $\tilde{D}_{4}$, which are the approximate and detail reconstructed subbands at level 4. During the decomposition, a target might be split into two different sub-bands if its frequency is situated in between two sub-bands. If this happens, it can potentially reduce the detection performance and we may consider the combination of $D_{3}$ and $D_{4}$ to produce a new reconstructed sub-band $\tilde{D}_{34}$.

Based on analysis of the Doppler spectra, it is proposed that $\tilde{D}_{123}, \tilde{A}_{4}, \tilde{D}_{4}$ and $\tilde{D}_{34}$ be used for the detection experiments; $\tilde{A}_{3}$ is omitted because it contains identical information to $\tilde{A}_{4}$ and $\tilde{D}_{4}$ combined. The PSD of these reconstructed sub-bands are shown in Fig. 6.
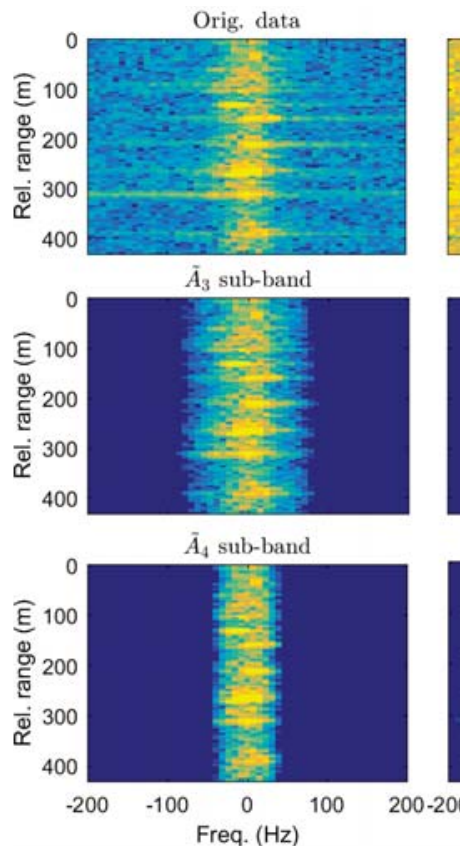

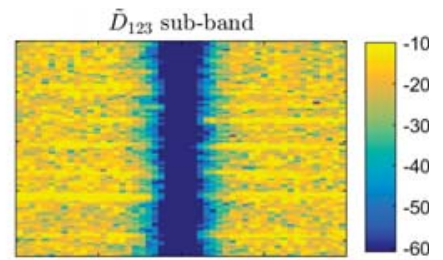
$\tilde{D}_{4}$ sub-band

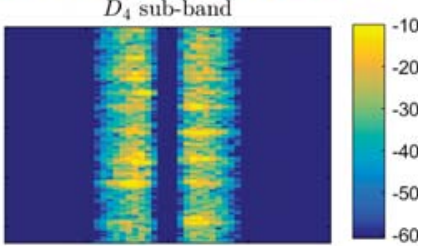

$\tilde{D}_{34}$ sub-band

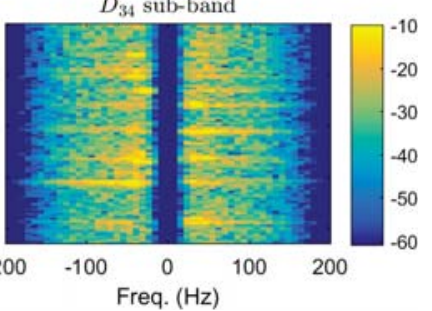

Fig. 6: PSD of the data after sub-band isolation and reconstruction using an SWT: $\tilde{A}_{3}$ and $\tilde{A}_{4}$ are the approximate reconstructed sub-bands of the original data at levels 3 and 4 respectively. $\tilde{D}_{123}$ is the combination of detail sub-bands $D_{1}, D_{2}$ and $D_{3}$ while the sub-band $\tilde{D}_{34}$ is the combination of $D_{3}$ and $D_{4}$.

\subsection{Sub-band indicator}

An entropy metric has been proposed in [15] to select the subbands which should provide the best detection performance. Entropy is a measure of information content and has been widely used to measure system disorder in statistical analysis [21], [22]. The motivation for using entropy is that a target is persistent over time, so its presence would alter the entropy measure compared to interference alone. Let $H(X)$ be the entropy of a discrete random variable, $X$, which models the samples in a range bin. It is found empirically when a target is present, $H(X)$ can be either less or greater than interference alone. To handle this ambiguity, we simply take the absolute value of its deviation from the global mean:

$$
E(X)=|H(X)-\langle H\rangle|,
$$

where $\langle H\rangle$ is the mean entropy over all range bins.

Rather than determine every possible reconstructed subband, a selection scheme is employed using the flowchart shown in Fig. 7 where $\gamma$ is the maximum entropy value of the range bins in a reconstructed sub-band: $\gamma=\max (E(X))$. The algorithm firstly compares the value of $\gamma_{\tilde{D}_{123}}$ and $\gamma_{\tilde{A}_{3}}$. If $\gamma_{\tilde{D}_{123}}$ is greater than $\gamma_{\tilde{A}_{3}}$, then $\tilde{D}_{123}$ is selected. Otherwise, the data is further decomposed and reconstructed to $\tilde{A}_{4}$ and $\tilde{D}_{4}$. A final entropy check then decides on either $\tilde{A}_{4}$ or the maximum of $\tilde{D}_{4}$ and $\tilde{D}_{34}$.

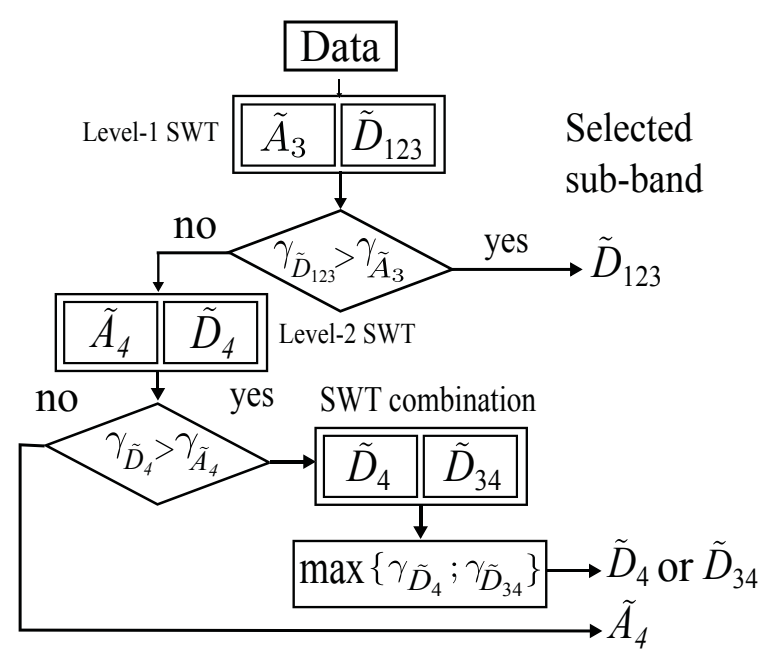

Fig. 7: Diagram of entropy sub-band indicator: the maximum entropy $(\gamma)$ is used to indicate the sub-band with the most information about the target.

\section{Detection analysis}

In maritime radars, target detection is typically performed with non-coherent processing due to the non-stationarity of the seaclutter. For this reason we compare the performance against unfiltered data in the range / time domain with application to the range / frequency domain left for future work. For the 
analysis here, a non-fluctuating (Swerling 0) target is used with variations of the target radial velocity $(0,1.9$ and 6.3 $\mathrm{m} / \mathrm{s}$ corresponding to Doppler frequency 0,31 and $102 \mathrm{~Hz}$ respectively). The target frequencies are chosen to be at the centre and edge of the endo-clutter region and out in the exo-clutter region. The data blocks used for comparison are horizontally polarised and includes bistatic angles of $60^{\circ}, 90^{\circ}$ and $120^{\circ}$. Each data block comprises a CPI of 128 pulses with the number of range bins dependent on the bistatic angle. Along with the probability of detection, $P_{\mathrm{d}}$, the proportion of reconstructed sub-bands is also shown for each result.

\subsection{Detection performance}

To investigate the detection performance using the SWT, a CA-CFAR algorithm is implemented along range to determine an adaptive threshold which adapts to the local clutter and maintains a constant false alarm rate. It is implemented with 32 range bins adjacent to the cell under test. To determine the threshold multiplier, a common technique is to run the CACFAR detection algorithm on the data with no target present. The multiplier is then varied to match the desired probability of false alarm, $P_{\mathrm{fa}}$, which is set to $10^{-3}$.

To determine the $P_{\mathrm{d}}$, a Monte Carlo simulation is implemented by repeatedly injecting targets at each range bin. The target SIR is varied and the detection scheme is run using the adaptive threshold determined by the CA-CFAR algorithm. The $P_{\mathrm{d}}$ is then determined by counting the number of detections which cross the threshold.

Figures 8 and 9 show the detection performance of the monostatic and bistatic data at a bistatic angle of $60^{\circ}$. The probability of detection for the three target velocities are shown in the first column as a function of the SIR with the number of times each sub-band is selected shown in the second column. The original (orig) non-filtered detection result is shown in blue, while the best reconstructed sub-band detection results are shown in red, green and black for the three target velocities. The detection result using the entropy indicator is plotted in magenta and denoted as 'eSWT'. For all detection comparisons, the required SIR is compared at $P_{\mathrm{d}}=0.5$.

For a stationary target, the best reconstructed sub-band is $\tilde{A}_{4}$, for a target moving with $1.9 \mathrm{~m} / \mathrm{s}$ (Doppler frequency of $31 \mathrm{~Hz}$ ), it is $\tilde{D}_{4}$ and with a velocity of $6.3 \mathrm{~m} / \mathrm{s}$ (Doppler frequency of $102 \mathrm{~Hz}$ ), the best result is found in $\tilde{D}_{123}$. When compared to the unfiltered data, the monostatic results show slightly worse performance for the eSWT due to the ineffectiveness of the indicator, while an increase of $0.6 \mathrm{~dB}$ is observed for the bistatic case. However when the target is moving, the eSWT algorithm shows greater improvement. For a slow target moving of $1.9 \mathrm{~m} / \mathrm{s}$, the improvement is $9.4 \mathrm{~dB}$ for the monostatic case and $11.6 \mathrm{~dB}$ for the bistatic. When the target moves faster, the eSWT shows an improvement of $20.9 \mathrm{~dB}$ and $14.3 \mathrm{~dB}$ for the monostatic and bistatic cases respectively.
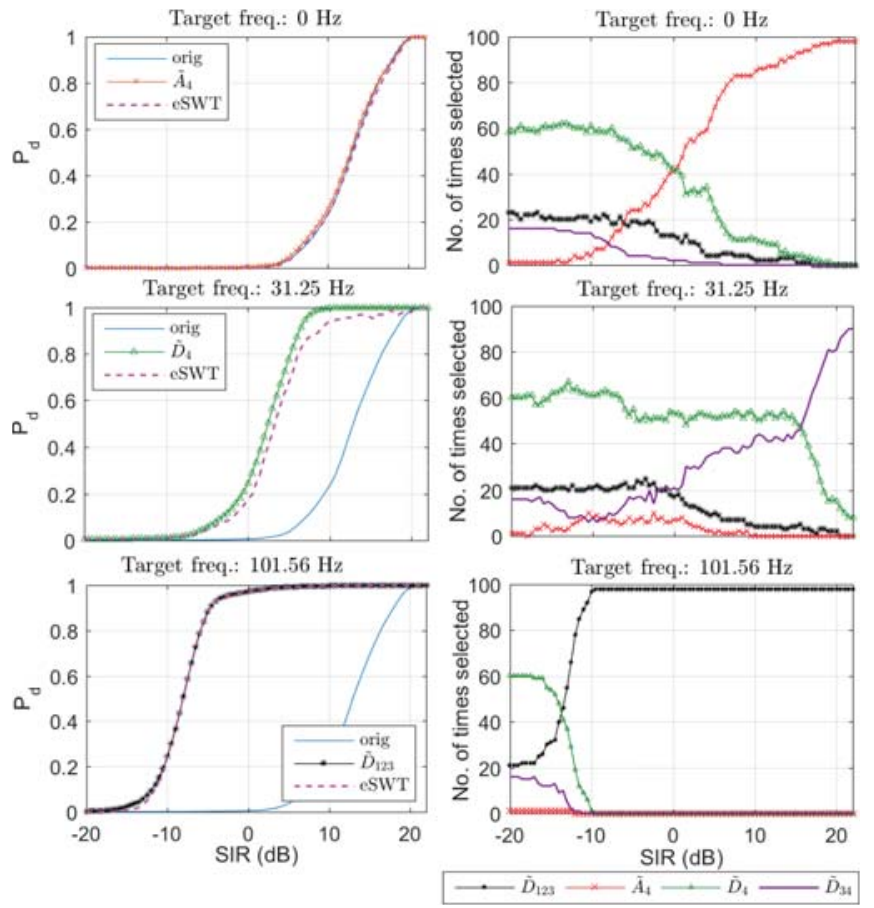

Fig. 8: NetRAD $60^{\circ}$ monostatic data: the probability of detection (first column) and the selected reconstructed subbands (second column)
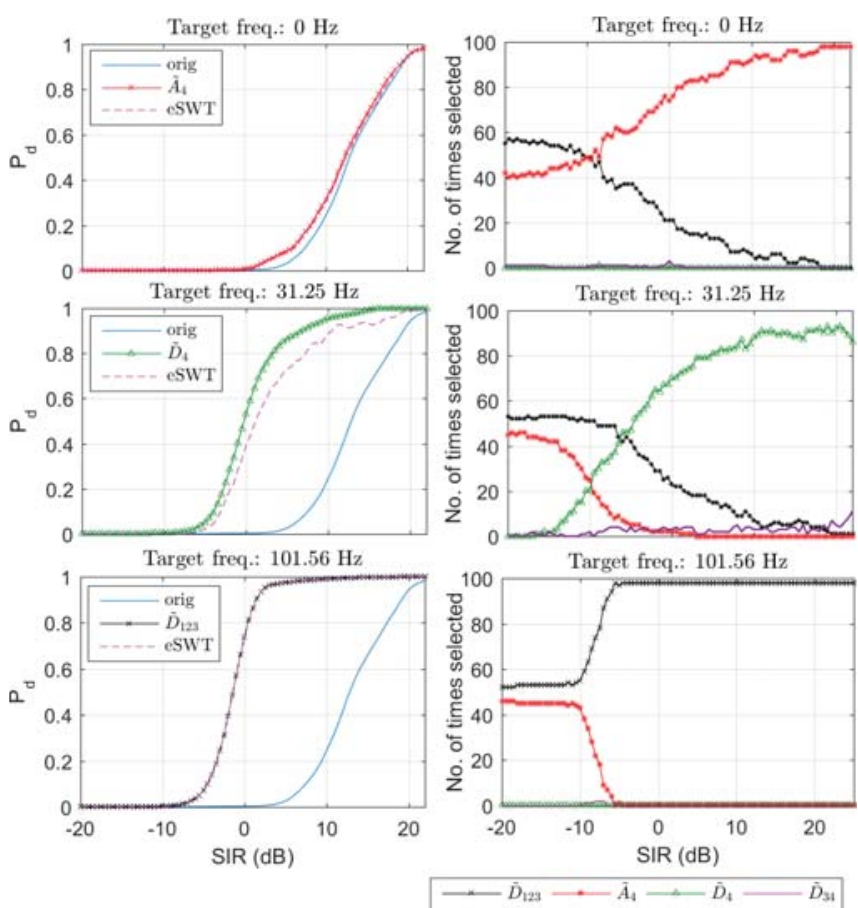

Fig. 9: NetRAD $60^{\circ}$ bistatic data: the probability of detection (first column) and the selected reconstructed sub-bands (second column). 
Table 1: SIR improvement relative to unfiltered data at $P_{\mathrm{d}}=0.5$ and $P_{\mathrm{fa}}=10^{-3}$.

\begin{tabular}{|c|c|c|c|c||c|c|c|}
\hline \multicolumn{2}{|c|}{ NetRAD } & \multicolumn{3}{|c||}{ Monostatic } & \multicolumn{3}{c|}{ Bistatic } \\
\cline { 3 - 8 } & $60^{\circ}$ & $90^{\circ}$ & $120^{\circ}$ & $60^{\circ}$ & $90^{\circ}$ & $120^{\circ}$ \\
\hline \hline \multirow{2}{*}{ Stat. } & $\tilde{A}_{4}$ & 0.1 & 0.5 & 1 & 0.6 & 0.2 & 0.5 \\
& eSWT & -0.1 & 0 & 0.6 & 0.4 & -1.3 & 0.2 \\
\hline \multirow{2}{*}{ Slow } & $\tilde{D}_{4}$ & 10.4 & 11.6 & 13.2 & 13.0 & 6.7 & 17.2 \\
& eSWT & 9.4 & 4.2 & 11.5 & 11.6 & 6.0 & 15.6 \\
\hline \multirow{2}{*}{ Fast } & $\tilde{D}_{123}$ & 20.9 & 18.1 & 19.0 & 14.3 & 12.5 & 19.6 \\
& eSWT & 20.9 & 18.1 & 19.0 & 14.3 & 12.1 & 19.6 \\
\hline
\end{tabular}

Table 1 summarises the performance improvement for all bistatic angles $\left(60^{\circ}, 90^{\circ}\right.$ and $\left.120^{\circ}\right)$ when compared to the unfiltered data. It is now clear that there is only a minor improvement between 0.1 to $0.6 \mathrm{~dB}$ for the stationary target, with the best results achieved using the $60^{\circ}$ bistatic data. For the slow moving target, the bistatic results typically have a larger improvement, with the maximum of $15.6 \mathrm{~dB}$ at a bistatic angle of $120^{\circ}$.

However, for the slow moving target using the monostatic $90^{\circ}$ data, there is a much smaller improvement in the eSWT when compared to the $\tilde{D}_{4}$ reconstructed sub-band. This is due to the entropy indicator selecting an incorrect reconstructed sub-band for the detection (i.e the reconstructed sub-band $\tilde{D}_{123}$ is selected for the detection instead of $\left.\tilde{D}_{4}\right)$. This confusion is caused by the interfering target as discussed in Section 2.2 .

\section{Conclusion}

This paper demonstrated a SWT-based detection scheme for small target detection in monostatic and bistatic sea-clutter. The process of sub-band isolation and reconstruction was illustrated with each reconstructed sub-band highlighting different features of the sea-clutter to better distinguish between targets and sea-spikes. Entropy was then used to select the appropriate reconstructed sub-band when the target velocity is not known prior to its detection.

To demonstrate the SWT scheme, a Monte-Carlo simulation using a CA-CFAR detection algorithm was implemented. The best reconstructed sub-bands with known target velocity was then compared with that determined by an entropy indicator. This analysis revealed that depending on the target velocity and bistatic angle, the improvement over unfiltered data varied between $-1.3 \mathrm{~dB}$ and $20.9 \mathrm{~dB}$. The entropy indicator was able to successfully determine the 'best' reconstructed sub-band in the majority of cases.

These results demonstrated that the SWT scheme can improve the detection performance in many cases. However, there is no clear indication whether a monostatic or bistatic configuration is more favourable. More trials and analysis are therefore required to clarify which geometry is preferable for the SWT detection approach. When compared with the Ingara medium grazing angle results shown in [15], the SWT detection scheme has proven robust and generally performs better for the NetRAD system when detecting moving targets.

\section{Acknowledgment}

The authors would like to thank Dr. Waddah A. Al-Ashwal for clarifying details of the NetRAD trial and data.

\section{References}

[1] K. Ward, R. Tough, and S. Watts, Sea Clutter: Scattering, the K Distribution and Radar Performance, 2nd ed. Institution of Engineering and Technology, 2013.

[2] M. Long, Radar Reflectivity of Land and Sea. Lexington Books, 2001.

[3] L. Rosenberg, "Sea-spike detection in high grazing angle X-band seaclutter," IEEE Transactions on Geoscience and Remote Sensing, vol. 51 no. 8, pp. 4556-4562, 2013.

[4] A. De Maio and M. S. Greco, Eds., Modern Radar Detection Theory. SciTech, 2016.

[5] V. Duk, B. Ng, and L. Rosenberg, "The potential of 2D wavelet transforms for target detection in sea-clutter," in IEEE Radar Conference, Arlington VA, May 2015, pp. 901-906.

[6] M. Rangaswamy, J. H. Michels, and B. Himed, "Statistical analysis of the non-homogeneity detector for STAP applications," Digital Signal Processing, vol. 14, no. 3, pp. 253 - 267, 2004.

[7] W. A. Al-Ashwal, "Measurement and modelling of bistatic sea clutter," PhD Thesis, Department of Electronic and Electrical Engineering University College London, 2011.

[8] W. A. Al-Ashwal, K. Woodbridge, and H. D. Griffiths, "Analysis of bistatic sea clutter-part II: amplitude statistics," IEEE Transactions on Aerospace and Electronic Systems, vol. 50, no. 2, pp. 1293-1303, 2014.

[9] F. Fioranelli, M. Ritchie, H. Griffiths, S. Sandenbergh, and M. Inggs, "Analysis of polarimetric bistatic sea clutter using the NetRAD radar system," IET Radar, Sonar \& Navigation, vol. 10, pp. 1356-1366, October 2016.

[10] M. Ritchie, A. Stove, K. Woodbridge, and H. G. Griffiths, "NetRAD: Monostatic and bistatic sea clutter texture and Doppler spectra characterization at S-band," IEEE Transactions on Geoscience and Remote Sensing, vol. 54, no. 9, pp. 5533-5543, September 2016.

[11] R. Palamà, M. Greco, and F. Gini, "Multistatic CFAR detection in nonGaussian clutter," EURASIP Journal on Advances in Signal Processing, vol. 2016, no. 107, 2016.

[12] N. Ehara, I. Sasase, and S. Mori, "Weak radar signal detection based on wavelet transform," in IEEE International Conference on Acoustics, Speech, and Signal Processing, vol. 2, Apr 1994, pp. 377-380.

[13] J. E. Ball and A. Tolley, "Low SNR radar signal detection using the continuous wavelet transform (CWT) and a Morlet wavelet," in IEEE Radar Conference, May 2008, pp. 1-6.

[14] S. Zhang, J. Fan, L. Shou, and J. Dong, "A detection method of radar signal by wavelet transforms," in Fourth International Conference on Fuzzy Systems and Knowledge Discovery, vol. 2, Aug 2007, pp. 710714.

[15] V. Duk, L. Rosenberg, and B. Ng, "Target detection in sea-clutter using stationary wavelet transforms," IEEE Transactions on Aerospace and Electronic Systems, vol. 53, no. 3, pp. 1136-1146, June 2017.

[16] J. S. Sandenbergh, M. R. Inggs, and W. A. Al-Ashwal, "Evaluation of coherent netted radar carrier stability while synchronised with GPSdisciplined oscillators," in IEEE Radar Conference, May 2011, pp. 1100-1105.

[17] W. A. Al-Ashwal, K. Woodbridge, and H. D. Griffiths, "Analysis of bistatic sea clutter-part I: average reflectivity," IEEE Transactions on Aerospace and Electronic Systems, vol. 50, no. 2, pp. 1283-1292, 2014.

[18] I. Daubechies, Ten Lectures on Wavelets. Philadelphia, PA, USA: Society for Industrial and Applied Mathematics, 1992.

[19] G. P. Nason and B. W. Silverman, "The stationary wavelet transform and some statistical applications," in Wavelets and Statistics. SpringerVerlag, 1995, pp. 281-300.

[20] N. Wang, Y. Zhang, and S. Wu, "Radar waveform design and target detection using wavelets," in CIE International Conference on Radar, 2001, pp. 506-509.

[21] K. F. Wallis, "A note on the calculation of entropy from histograms," Review Literature And Arts Of The Americas, October 2006.

[22] B. Harris, "Entropy," John Wiley and Sons, Inc, 2006. 